\title{
A call to action: Addressing the reproductive health needs of women with drug-resistant tuberculosis
}

\author{
K Schnippel, N Ndjeka, F Conradie, R Berhanu, Z Claasen, S Banoo, C Firnhaber \\ Kathryn Schnippel is Senior Epidemiologist at Right to Care, Johannesburg, South Africa, and is also Senior Researcherat the Clinical HIV Research Unit, \\ School of Clinical Medicine, Faculty of Health Sciences, University of the Witwatersrand, Johannesburg. Norbert Ndjeka is Director: Drug-Resistant \\ TB, TB \& HIV at the National TB Control \& Management Cluster, National Department of Health, South Africa. Francesca Conradie is MDR-TB \\ Technical Advisor at Right to Care and Clinical Research Advisor, Clinical HIV Research Unit. Rebecca Berhanu is Infectious Disease Fellow at the \\ Department of Medicine, Division of Infectious Diseases, University of North Carolina, USA. Zerilda Claasen is Technical Advisor, Drug-Resistant \\ TB, TB \& HIV, National TB Control \& Management Cluster. Shabir Banoo is Head: Pharmaceutical Policy at the Research and Services Support Unit, \\ Right to Care. Cynthia Firnhaber is Associate Professor in Internal Medicine, Clinical HIV Research Unit, and the Head of Research at Right to Care.
}

Corresponding author: K Schnippel (kschnipp@gmail.com)

\begin{abstract}
Although there is substantial risk to maternal and neonatal health in the situation of pregnancy during treatment for rifampicin-resistant tuberculosis (RR-TB), there is little evidence to guide clinicians as to how to manage this complexity. Of the 49680 patients initiated on RR-TB treatment from 2009 to 2014 in South Africa, 47\% were women and 80\% of them were in their reproductive years (15 - 44). There is an urgent need for increased evidence of the safety of RR-TB treatment during pregnancy, increased access to contraception during RR-TB treatment, and inclusion of reproductive health in research on the prevention and treatment of TB.
\end{abstract}

S Afr Med J 2016;106(4):333-334. DOI:10.7196/SAMJ.2016v106i4.10205

An analysis of the global burden of tuberculosis (TB) during pregnancy estimated that each year, more than 8400 pregnant women in South Africa (SA) have active TB. ${ }^{[1]}$ To put this burden into context, across sub-Saharan Africa, the combination of TB and HIV in pregnancy is a leading cause of maternal mortality ${ }^{[2]}$ and in SA an estimated $65 \%$ of TB patients are HIV-positive. SA also ranks among the top countries in the world in terms of the number of TB patients with rifampicinresistant TB (RR-TB), ${ }^{[3]}$ including multidrug-resistant (MDR) TB and extensively drug-resistant (XDR) TB. RR-TB requires 18 - 24 months of complex treatment with a high frequency of adverse drug reactions and poor treatment outcomes. Globally less than $50 \%$ of the 2011 cohort of MDR-TB patients were treated successfully. ${ }^{[3]}$

Globally, there is scant evidence on the safety of second-line TB medications during pregnancy. Clinicians rely on data extrapolated from decades-old clinical trials ${ }^{[4]}$ or from limited case series. ${ }^{[5]}$ Clinicians must weigh the scant safety data against evidence that delayed RR-TB treatment may increase the risk of obstetric and neonatal complications from TB disease ${ }^{[6]}$ ongoing transmission, and that suboptimal RR-TB treatment can lead to failure and the amplification of resistance.

\section{Estimating the burden of pregnancy during $\mathbb{R} R-T B$ treatment in SA}

In order to quantify the risk of pregnancy and RR-TB treatment, we analysed the drug-resistant TB case registry in SA to assess the number of women receiving RR-TB during their reproductive years.

\section{Setting and treatment guidelines}

SA RR-TB patients receive a standardised regimen: 6 months of an injectable drug (kanamycin or amikacin) and 18 - 24 months of moxifloxacin, ethionamide, terizidone and pyrazinamide. Patients with second-line drug resistance, toxicity, or contraindications to the standardised regimen can access individualised regimens including capreomycin, para-aminosalicylic acid, clofazimine, linezolid and bedaquiline. SA has universal testing for rifampicin resistance with public sector coverage of Xpert MTB/RIF since the end of 2013.

\section{Data extraction and analysis}

The Electronic DR-TB Register (EDRweb) is the RR-TB national case register and, since 2012, has been used for reporting to the World Health Organization (WHO). A census of patients registered in the EDRweb from January 2009 to December 2014 was exported ( $N=53623$ ). Registrations missing sex, report of rifampicin resistance or date of treatment initiation details were excluded. Pregnancy and pregnancy outcomes are not routinely reported in the EDRweb.

Descriptive counts and proportions for gender, age category (0 - 14, 15 - 29, 30 - 44, 45 - 59, 60+), HIV status (negative, positive or unknown), history of prior TB treatment with any outcomes, and category of drug resistance (RR-TB and MDR-TB or secondline drug resistance as indicated by XDR-TB case registration) are presented. Differences of proportions were estimated using Pearson's $\chi^{2}$ and $p$-values are presented. All analyses were conducted in Stata SE version 13 (College Station, USA).

\section{Ethical approval}

The University of the Witwatersrand Human Research Ethics Committee approved this retrospective, de-identified, secondary analysis in April 2015.

\section{Results}

From 2009 to 2014, 49680 patients with RR-TB were registered and initiated on second-line TB treatment in the EDRweb. Nearly half $(47.2 \%, n=23428)$ were female. Males had a median age of 37 (interquartile range (IQR) 30 - 45) years and females a median age of 32 (IQR $26-40)$ years. Eighty percent $(n=18750)$ of the women treated for RR-TB were aged 15 - 44 years.

At initiation of RR-TB treatment (Table 1), women were younger $(40.0 \%$ v. $24.9 \%$ aged $<30)$, more likely to be HIV-infected $(68.0 \%$ v. 
$57.2 \%$ ), less likely to have a history of TB treatment prior to their RR-TB diagnosis $(60.8 \%$ v. $65.7 \%)$, and more likely to have XDR-TB (6.9\% v. 6.2\%) than men. Women aged 15 - 44 years were more likely to be HIVpositive than women $<15$ years or $>44$ years (70.8\% v. $53.9 \%, p<0.000)$.

\section{An urgent call to action}

From 2009 to 2014, 23428 women initiated RR-TB treatment in SA. Of these women on second-line TB treatment, $80 \%$ were of reproductive age. In contrast, a 2012 review of TB treatment in pregnant and postpartum women found only eight published case reports to guide second-line TB treatment during pregnancy, describing outcomes for 73 women. ${ }^{[5]}$ Most (68.0\%) of the women treated for RR-TB in SA are also HIV-positive, yet the case reports describe outcomes for just six HIV-positive women. ${ }^{[5]}$

These numbers should serve as an urgent call to action:

\section{Collect more evidence of the safety of second-line TB treatment during pregnancy}

Mothers with RR-TB and their clinicians are faced with a difficult decision. Should they treat the mother's RR-TB and expose the fetus to potentially toxic drugs, or delay or stop treatment and face the risk of maternal or fetal mortality or the amplification of drug resistance? The principal concern is ototoxicity related to the injectable drugs, among the mainstays of second-line TB treatment. More evidence of the safety of second-line TB treatment during pregnancy is urgently needed to inform these decisions. This is a broader call than adverse drug event monitoring given the importance of also reporting the outcomes of TB and pregnancy even in the absence of adverse drug events. Monitoring RR-TB and pregnancy outcomes requires co-ordination across different facilities and health directorates, as women need to be identified during antenatal care and followed through delivery and postpartum. The drugs-exposure pregnancy register led by the WHO can serve as a model of a feasible system for resourcelimited contexts. ${ }^{[7]}$

\section{Ensure access to effective contraception during second-line TB treatment}

Currently, contraception services in SA are offered at primary healthcare level while RR-TB services are more centralised, often still at hospital level. Offering effective contraception to women on second-line TB treatment could reduce the number of pregnancies during active TB. Integration of

Table 1. Differences in baseline characteristics of RR-TB patients registered in the EDRweb, 2009 - 2014, by sex

\begin{tabular}{lllll}
\hline & Female, $\boldsymbol{n}(\%)$ & Male, $\boldsymbol{n}(\%)$ & Total, $\boldsymbol{n}(\%)$ & $\boldsymbol{p}$-value \\
\hline All RR-TB & $23428(47.2)$ & $26242(52.8)$ & $49680(100)$ & \\
Age (years) & & & & $<0.000^{*}$ \\
$0-14$ & $768(3.3)$ & $584(2.2)$ & $1352(2.7)$ & \\
$15-29$ & $8597(36.7)$ & $5994(22.7)$ & $14541(29.3)$ & \\
$30-44$ & $10153(43.3)$ & $12575(47.9)$ & $22728(45.7)$ & \\
$45-59$ & $3233(13.8)$ & $6107(23.3)$ & $9340(18.8)$ & $<0.000$ \\
$\geq 60$ & $687(2.9)$ & $1032(3.9)$ & $1719(3.5)$ & \\
HIV infection & & & $13677(27.5)$ & \\
HIV-negative & $5288(22.6)$ & $8389(32.0)$ & $30947(62.3)$ & \\
HIV-positive & $15936(68.0)$ & $15011(57.2)$ & $5056(10.2)$ & \\
HIV unknown & $2214(9.4)$ & $2842(10.8)$ & & \\
Previous TB & & & $18124(36.6)$ & \\
No prior TB & $9158(39.2)$ & $8966(34.3)$ & $31378(63.4)$ & \\
History of TB & $14191(60.8)$ & $17187(65.7)$ & & \\
Drug resistance & & & &
\end{tabular}

family planning services within the RR-TB units is therefore urgently needed.

\section{Ensure women's health is included in research on prevention and treatment of TB}

Effective TB research is the third pillar of the WHO strategy to end TB. This research must address women's health, especially reproductive health. More information and evidence on effective TB prophylaxis for women and infants, pharmacokinetics of TB drugs in pregnancy and pharmacokinetics of contraceptives and antiretrovirals during TB treatment is needed urgently. ${ }^{[8]}$

\section{Conclusion}

In SA, where the burden of RR-TB and TB/ HIV co-infection is high, there is an urgent need for increased evidence of the safety of RR-TB treatment during pregnancy. Increased access to contraception during RR-TB treatment and inclusion of reproductive health in research on the prevention and treatment of TB need to be addressed.

Acknowledgements. The analysis presented here is based on data collected by the SA Department of Health and extracted with the support of S'celo Dlamini and Nontobeko Msthali.

Funding. N Ndjeka is responsible for the DRTB programme within the SA National TB Programme. K Schnippel, F Conradie, R Berhanu,
S Banoo and C Firnhaber were supported through USAID cooperative agreement \#674-A-12-00020 to Right to Care. Z Claasen is supported by the Lilly Foundation grant to FHI360. The content of the article is the responsibility of the authors and does not necessarily reflect the views of the SA government, USAID or the US government. The funders had no role in the study design, collection, analysis and interpretation of the data, in manuscript preparation or in the decision to publish.

\footnotetext{
1. Sugarman J, Colvin C, Moran AC, Oxlade O. Tuberculosis in pregnancy: An estimate of the global burden of disease. in pregnancy: An estimate of the global burden of disease.
Lancet Glob Health 2014;2(12):e710-716. DOI:10.1016/S2214109X(14)70330-4

2. Grange J, Adhikari M, Ahmed Y, et al. Tuberculosis in association with HIV/AIDS emerges as a major nonobstetric cause of maternal mortality in Sub-Saharan Africa. Int J Gynaecol Obstet 2010;108(3):181-183. DOI:10.1016/j.ijgo.2009.12.005

3. World Health Organization (WHO). Global Tuberculosis Report 2014. Geneva: WHO, 2014. http://www.who.int/tb/ publications/global_report/en/ (accessed 15 November 2015)

4. Briggs GG, Freeman RK, Yaffe SJ. Drugs in Pregnancy and Lactation: A Reference Guide to Fetal and Neonatal Risk. 9th ed. Philadelphia: Lippincott Williams \& Wilkins, 2011:1703.

5. Mathad JS, Gupta A. Tuberculosis in pregnant and postpartum women: Epidemiology, management, and research gaps. Clin women: Epidemiology, management, and research gaps. Clin

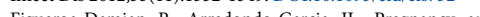

Figueroa-Damian R, Arredondo-Garcia JL. Pregnancy and tuberculosis: Influence of treatment on perinatal outcome. Am

Perinatol 1998,15(5)

7. Mehta U, Clerk C, Allen E, et al. Protocol for a drugs exposure pregnancy registry for implementation in resourcelimited settings. BMC Pregnancy Childbirth 2012;12(1):89. DOI:10.1186/1471-2393-12-89

8. Mcllleron H, Abdel-Rahman S, Dave JA, Blockman M, Owen A. Special populations and pharmacogenetic issues in tuberculosis drug development and clinical research. J Infect Dis 2015;211(Suppl 3):S115-S125. DOI:10.1093/infdis/jiu600
}

Accepted 23 October 2015. 